\title{
Magnetic Field Effects on Cytochrome c-Mediated Bioelectrocatalytic Transformations
}

\author{
Eugenii Katz, Oleg Lioubashevski and Itamar Willner* \\ Institute of Chemistry, The Hebrew University of Jerusalem, Jerusalem \\ 91904, Israel
}

\section{Supporting Information}

The model presented in ref. 12 formulated the relation between the limiting current density, $i_{\mathrm{L}}$, the magnetic field strength, $B$, and the substrate bulk concentration, $C^{*}$, as given by Equation $\mathrm{X}$ :

Eq. X: $\quad i_{\mathrm{L}}=0.62(\rho R)^{-1 / 3} D^{8 / 9} v^{-2 / 9}\left(n F C^{*}\right)^{4 / 3} B^{1 / 3}$

where $n$ is the number of the electrons involved in the Faradaic process, $F$ is the Faraday's number, $R$ is an electrode characteristic size, $\rho$ and $v$ are the fluid specific density and kinematic viscosity, respectively, $D$ and $C^{*}$ are the diffusion coefficient and the bulk concentration of the redox species, respectively, and $B$ is magnitude of the imposed magnetic field.

The proportionality factor between $i_{\mathrm{L}}$ and $B^{1 / 3} C^{4 / 3}$ reveals very good agreement between the calculated and observed values (Table 1) for systems that include simple low molecular weight redox-active species (corresponding to the systems described in ref. 6e and ref. $6 \mathrm{f}$ ). For the present system, however, a sigificant deviation in the proportionality factor between $i_{\mathrm{L}}$ and $B^{1 / 3} C^{4 / 3}$ is observed. This may originate from the fact that the electron transfer that involves cytochrome $\mathrm{c}$ includes the equilibrium association and disociation of the protein to and from the receptor sites linked to the electrode surface. This phenomenon is not included in the quatitative formulation of the theoretical model. 
Table 1.

Comparison of the experimental and calculated limiting current densities.

\begin{tabular}{|c|c|c|c|c|c|c|c|c|}
\hline Ref. & $\begin{array}{c}\boldsymbol{C}^{*}, \\
\mathbf{m M}\end{array}$ & $\begin{array}{c}\boldsymbol{B}, \\
\mathbf{T}\end{array}$ & $\begin{array}{c}\boldsymbol{i}_{\text {experimental }} \\
\mathbf{A} \cdot \mathbf{m}^{-2}\end{array}$ & $\begin{array}{c}\boldsymbol{i}_{\text {calculated }} \\
\mathbf{A} \cdot \mathbf{m}^{-2}\end{array}$ & $\begin{array}{c}\mathbf{D}, \\
\mathbf{c m}^{2} \cdot \mathbf{s e c}^{-\mathbf{1}}\end{array}$ & $\begin{array}{c}\boldsymbol{v}, \\
\mathbf{c m}^{2} \cdot \mathbf{s e c}^{-\mathbf{1}}\end{array}$ & $\begin{array}{c}\rho, \\
\mathbf{g} \cdot \mathbf{c m}^{-3}\end{array}$ & $\begin{array}{c}\boldsymbol{R}, \\
\mathbf{m}\end{array}$ \\
\hline Ref. 6f & 19.9 & 1.75 & 60.1 & 109 & $2.42 \times 10^{-5}$ & $6.27 \times 10^{-3}$ & 0.826 & $8 \times 10^{-4}$ \\
\hline & 31.8 & 1.75 & 113.7 & 203 & $2.42 \times 10^{-5}$ & $6.27 \times 10^{-3}$ & 0.826 & $8 \times 10^{-4}$ \\
\hline Ref. 6e & 2000 & 0.4 & $2.85 \times 10^{4}$ & $7.7 \times 10^{4}$ & $1.8 \times 10^{-5}$ & $6.27 \times 10^{-3}$ & 0.826 & $2.5 \times 10^{-5}$ \\
\hline & 2000 & 0.6 & $3.26 \times 10^{4}$ & $8.7 \times 10^{4}$ & $1.8 \times 10^{-5}$ & $6.27 \times 10^{-3}$ & 0.826 & $2.5 \times 10^{-5}$ \\
\hline Present & 0.1 & 0.92 & 0.11 & 0.0027 & $1.04 \times 10^{-6}$ & 0.01 & 1 & $2.5 \times 10^{-3}$ \\
paper & & & & & & & & \\
\hline
\end{tabular}

